Int. J. Dev. Biol. 55: 851-859

doi: $10.1387 / \mathrm{ijdb} .113365 \mathrm{jt}$

\title{
Adipocyte is a non-trivial, dynamic partner of breast cancer cells
}

\author{
JINXIANG TAN ${ }^{1, \#,}$ EMILIE BUACHE $1, \#$, MARIE-PIERRE CHENARD ${ }^{2}$, \\ NASSIM DALI-YOUCEF ${ }^{1,3}$, MARIE-CHRISTINE RIO*,1
}

\begin{abstract}
${ }^{1}$ Institut de Génétique et de Biologie Moléculaire et Cellulaire (IGBMC), Functional Genomics and Cancer Department, Centre National de la Recherche Scientifique UMR 7104, Institut National de la Santé et de la Recherche Médicale U964, Université de Strasbourg, France, ${ }^{2}$ Centre Hospitalier Universitaire de Hautepierre, Département de Pathologie and ${ }^{3}$ Laboratoire de Biochimie et de Biologie Moléculaire, Hôpitaux Universitaires de Strasbourg, Strasbourg, France
\end{abstract}

\begin{abstract}
While the participation of adipocytes is well known in tissue architecture, energy supply and endocrine processes, their implication during natural cancer history is just beginning to unfold. An extensive review of the literature concerning the impact of resident adipocytes on breast cancer development/progression was performed. This review provides in vitro and in vivo evidence that adipocytes located close to invasive cancer cells, referred to as cancer-associated adipocytes (CAAs), are essential for breast tumor development/progression. Their deleterious function is dependent, at least partly, on their crosstalk with invasive cancer cells. Indeed, this event leads to dramatic phenotypic and/or functional modifications of both cell types. Adipocytes exhibit delipidation and acquire a fibroblast-like shape. In parallel, cancer cell aggressiveness is exacerbated through increased migratory and invasive properties. Moreover, obesity is currently a sign of poor prognosis in human carcinomas. In this context, a high number of "obese" resident adipocytes might be predicted to be detrimental. Accordingly, there are some similarities between the molecular alterations observed in hypertrophied adipocytes and in CAAs. How adipocytes function to favor tumorigenesis at the molecular level remains largely unknown. Nevertheless, progress has been made recently and molecular clues are starting to emerge. Deciphering the ceIlular and molecular mechanisms behind the adipocyte-cancer cell heterotypic crosstalk is of great interest since it might provide new targets for improving diagnosis/prognosis and for the design of innovative therapeutic strategies. They might also improve our understanding of the relationship between obesity/metabolic disorders and cancer risk and/or poor patient outcome.
\end{abstract}

KEY WORDS: adipocyte, breast cancer, metabolism, obesity, diabetes

\section{Introduction}

It is now well established that the tumor microenvironment actively participates in breast cancer progression (Basset et al., 1990; Jodele et al., 2006; Kessenbrock et al., 2010). The connective tissue, also named stroma, is a complex structure composed of an extracellular matrix (ECM), and a variety of cell types including endothelial cells, inflammatory cells, fibroblasts, fibroblast-like cells and adipocytes. The present review is restricted to the latter cell type, and more precisely to the impact of mammary gland resident adipocytes on breast cancers. The first part focuses on cancerassociated adipocytes (CAAs) as local regulators of epithelial cancer cell growth, local invasion and/or metastases in carcinogenesis. Although the nature of interplay between adipocytes and cancer cells remains at present largely unknown, investigations in the field over the last 10 years indicate that the adipocyte occupies a central place in this disease. The morphological and functional alterations

\footnotetext{
Abbreviations used in this paper: $\mathrm{Ad}$, adipocytes cultured alone; $\mathrm{Ad}_{\mathrm{BCC}}$, adipocytes cultured in the presence of breast cancer cells; $\mathrm{BCC}$, breast cancer cells cultured alone; $\mathrm{BCC}_{\mathrm{Ad}}$, breast cancer cells cultured in the presence of adipocytes; CAA, cancerassociated adipocytes; CAF, cancer-associated fibroblasts; ECM, extracellular matrix; ER, estrogen receptors; MMP, matrix metalloproteinase.
}

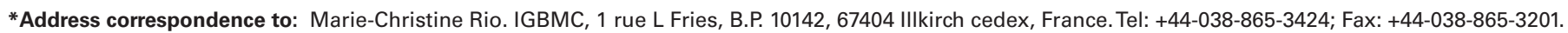
e-mail: rio@igbmc.fr - web: http://www-igbmc.fr \# Note: These authors contributed equally to this work.
}

Final, author-corrected PDF published online: 2 September 2011. 


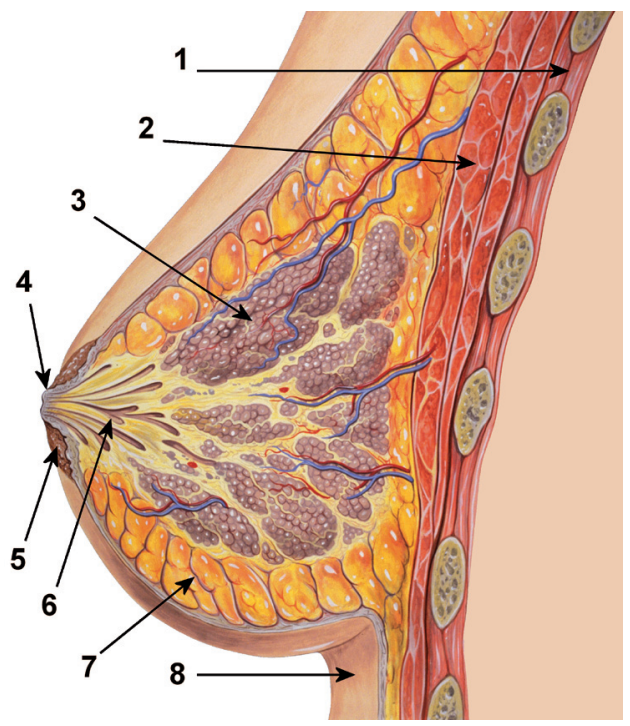

Fig. 1. Schematic diagram of normal breast (adult female human cross-section, Wikipedia). 1, chest wall; 2, pectoralis muscles; 3, lobules; 4, nipple; 5, areola; 6, duct; 7 fatty tissues; 8, skin. Resident adipocytes represent one of the most prominent cell types of the mammary gland.

in both adipocytes and cancer cells induced by the CAA-cancer cell heterotypic crosstalk, are discussed, as well as the emerging molecular basis that underlies the pejorative effect of CAAs on the behavior of cancer cells. The second part focuses on how adipocyte alterations due to metabolic diseases (e.g. obesity, diabetes) might impact the development and progression of breast cancers. A thorough understanding of the role of adipocytes in mammary gland tumorigenesis could present numerous opportunities to improve tumor prognosis, to design innovative therapies and/or to modify breast cancer risk.

\section{Cancer-associated adipocyte (CAA)-cancer cell cross- talk favors breast tumor progression}

\section{The role of adipocytes in the normal breast}

The mammary gland is a dynamic organ that continually changes its architecture and function. Reciprocal interactions between the epithelium and the connective tissues exert profound effects on mammary gland morphogenesis, development and homeostasis, even though the details of these events are not fully understood. The normal development and function of the mammary gland are reviewed elsewhere in the present issue. Here only a brief overview of the anatomy of the human adult mammary gland is given in order to better understand the particular place of the adipocyte in breast tissues. The fully differentiated breast (after the first gestation-lactation cycle) is composed of two cell compartments (Fig. 1). The epithelial compartment is constituted by the gland (strickly speaking) composed of branched ducts and lobuloalveolar differentiated units able to produce milk proteins when necessary. This gland is embedded in the connective tissue compartment commonly referred to as the mammary fat pad (Hovey and Aimo, 2010) since it mainly contains adipose tissues. In man, the mammary adipose depot is composed of fat lobules involving 100-1000 mature white adipocytes and stromal-vascular cells, blood vessels, lymph nodes and nerves.

In a general manner, each adipocyte is itself encapsulated by a basement membrane. Once cells are committed pre-adipocytes, the original ECM is remodeled. Indeed, adipogenesis is characterized by the conversion of a fibronectin-rich matrix to a basement membrane (Gregoire, 2001). During adipocyte differentiation, there is an increase in the secretion of basement membrane components such as type V and VI collagens (Nakajima et al., 2002) (lyengar et al., 2005). Thus, in a general fashion, normal mature adipocytes are separated from epithelial cells by basement membranes, limiting therefore the possibilities of heterotypic cross-talk between the 2 cell types. In the normal breast, this equilibrium is dramatically disrupted during the postweaning mammary gland involution. At this time, there is an intense restructuration of the gland and cells of the 2 compartments are mixed together within the ECM, as observed in invasive primary tumors (Lefebvre et al., 1992).

Thus, fat tissue represents an important component of the normal breast. This obvious proximity of adipocytes and epithelial cells in the breast allows one to hypothesize that adipocytes might play a role during tumor progression.

\section{Histological evidence that adipocytes participate in breast cancer}

The role of adipocytes during cancer invasive processes has until recently been neglected. This lapse was mainly due to the fact that adipocytes disappear rapidly via the desmoplastic response of connective tissues during the early invasive steps. Thus, histological images of breast tumor biopsies contain very few adipocytes, or most often are totally devoid of adipocytes. However, from the breast anatomy (see above and Fig. 1), it might be expected that early local tumor invasion occurs in the immediate proximity of pre-adipocytes and/or adipocytes, allowing heterotypic cross-talk between invasive cancer cells and adipocytes, even if this event is ephemeral. In this context, histological sections of human breast carcinomas show that dynamic desmoplastic events involving adipocytes occur at the tumor invasive front located at the periphery of primary tumors (Fig. 2) (Andarawewa et al., 2005) (Dirat et al., 2011). Indeed, this area is devoid of fully constituted stroma and exhibits a high ratio

Fig. 2. Histology of the invasive front and the center of a human breast carcinoma. Hematoxylin-eosin histological examination. (A) The invasive front of a primary tumor. Features of connective tissues are dramatically modified in response to cancer cell local invasion. Most importantly, CAAs (lipids in white color) located at the interface of invading cancer cells exhibit reduced size in comparison with normal adipocytes located at a distance; (B) The center of the same tumor shows accumulation of fibroblasts/fibroblast-like cells (CAFs), and adipocytes are no longer present.
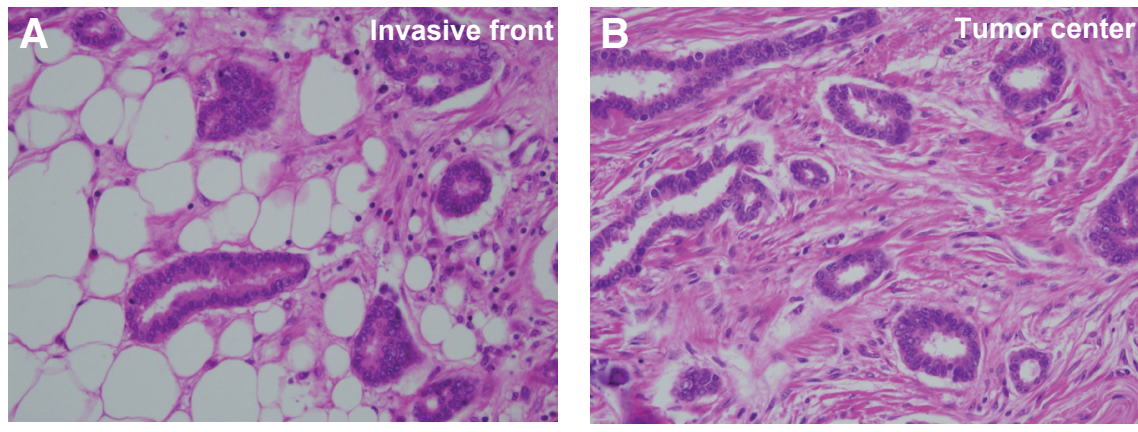
of adipocytes to fibroblasts. Interestingly, a reduction in adipocyte size compared with those observed at a distance is observed. This size reduction of adipocytes implies lipolysis, modification of lipid droplets and modification of adipocyte-related basement membrane and the ECM. By contrast, at the tumor center, an extremely high fibroblast-like cell:adipocyte ratio is observed in the stroma surrounding cancer cells. Thus, one of the most obvious morphological changes that occur consecutively to cancer cell invasion concerns adipocytes, pointing-out the notion of CAAs. It is therefore clear that CAA-cancer cell heterotypic crosstalk occurs, at least in invasive primary tumors, when both cell types are physically close during the dynamic tumor progression processes. This event leads to a kind of "adipocyte dedifferentiation" and ultimately to an accumulation of fibroblast-like cells. Tumor progression may thus depend on the CAA "activation" induced by invading cancer cell stimuli. Moreover, these data strongly support the concept that cancer-associated fibroblasts (CAFs) might at least partly derive from CAAs (Andarawewa et al., 2005; Motrescu and Rio, 2008). Interestingly, numerous studies have shown that peritumor fibroblasts provide structural and biochemical support for cancer development (Ronnov-Jessen et al., 1995)(Kessenbrock et al., 2010).

Thus, the adipocyte is an excellent candidate to play a key role in influencing tumor behavior through heterotypic crosstalk with invasive cancer cells. In turn, signaling pathways critical for cancer cell survival, tumor growth and/or metastasis development may be inadvertently activated.

\section{In vivo evidence that adipocytes favor tumor progression}

Does CAA-cancer cell cross-talk have a pejorative impact on tumor progression? Numerous recent clinical studies have evaluated the prognosis value of local adipose tissue invasion by cancer cells at the tumor margin. Most of the data report a positive correlation with poor patient outcome. This is the case not only for breast cancers (Kimijima etal., 2000; Yamaguchi et al., 2008), but also for prostate, pancreas, kidney and colon cancers (Rio, 2011). Experimental data report a similar pejorative impact of adipocytes on cancer progression. It was shown that an adipocyte-rich environment facilitates SPI murine mammary carcinoma cell growth after subcutaneous injection in mice, and it was proposed that the adipose tissue exerts an estrogen-dependent positive regulatory effect on SPI (Elliott et al., 1992). The impact of the mammary fat pad in tumor development has also been extensively studied (Edwards, 2000). Moreover, adipose stromal cells have been shown to stimulate the migration and invasion of estrogen receptor (ER)-negative breast cancer cells in vitro and in vivo in a co-transplant xenograft mouse model. However, it should be noted that both the mammary fat pad and adipose stromal cells include both pre-adipocytes and/or adipocytes, as well as other cells like endothelial cells, that may interfere. An in vivo coinjection system using 3T3-L1 murine adipocytes and SUM159PT human breast cancer cells to recapitulate heterotypic crosstalk occuring in primary breast tumors has demonstrated that adipocytes favor tumorigenesis (lyengar et al., 2003). Using a similar model, it has recently been shown that adipocytes also favor metastasis development. Indeed, tail vein injection of breast cancer cells previously co-cultured in the presence of adipocytes gives more lung metastases than breast cancer cells grown without adipocytes (Dirat et al., 2011).

Thus, clinical observations and in vivoexperimental data strongly support a major function for mammary gland resident adipocytes in tumor progression.

\section{In vitro evidence that adipocytes favor cancer cell aggres- siveness}

Because in vivo, several other local or systemic factors might interfere, in vitro cell models have been designed to uncover the intrinsic properties and functions of adipocytes in breast cancer. The relative influence of pre-adipocytes and mature adipocytes on various cancer cell lines have been studied by several laboratories. Mature adipocytes, but not pre-adipocytes, have been shown to promote the growth of breast cancer cells in collagen gel matrix cultures through cancer-stromal cell interactions (Manabe et al., 2003). More recently, a co-culture system in which adipocytes and breast cancer cells were separated by an insert to allow the diffusion of soluble factors, but not direct heterotypic cell-cell contact, was used to mimic the CAA-cancer cell crosstalk. The impact of this co-culture on both adipocytes and cancer cells was evaluated. As expected from previous histological studies (see above), dramatic morphological changes were observed in adipocytes co-cultured in the presence of breast cancer cells $\left(\mathrm{Ad}_{\mathrm{BCC}}\right)$ compared with those cultured alone (Ad). As observed at the invasive front of breast primary tumors, adipocytes became more elongated and exhibited a fibroblast-like phenotype with delipidation (Fig. 3). There was no obvious alteration in cancer cell morphology. However, adipocyteprimed cancer cells $\left(\mathrm{BCC}_{\mathrm{Ad}}\right)$ aquired new properties, notably an increased migration and invasive phenotype compared with cancer cells that had not "seen" adipocytes (BCC) (Fig. 4) (Dirat etal., 2011) (our unpublished results). Thus, this model satisfactorily recapitulates the invasive front of human breast tumors. These data indicate that soluble factors resulting from the adipocyte-cancer cell crosstalk impact both cell types. Indeed, adipocyte-derived secreted factors have been shown to promote breast cancer cell proliferation and

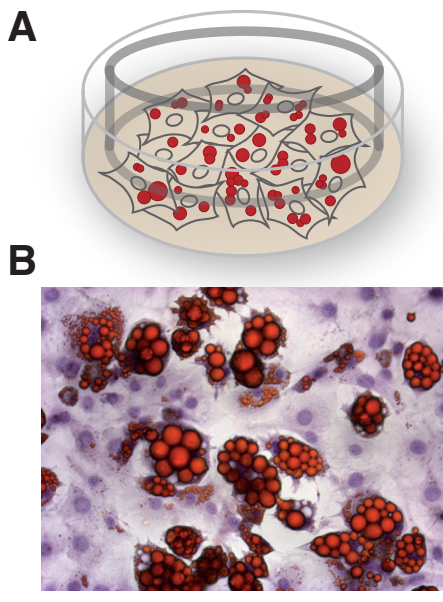

Ad
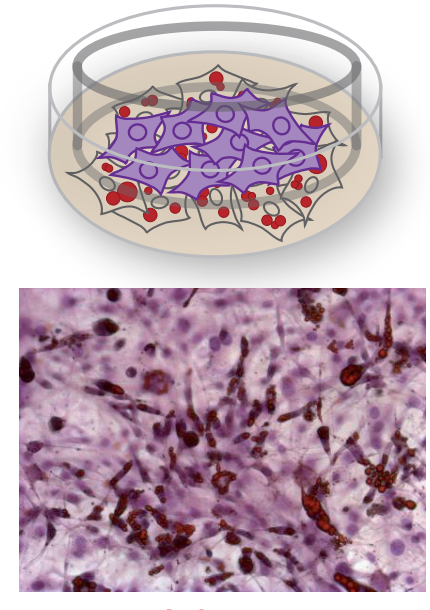

$\operatorname{Ad}_{\mathrm{BCC}}$
Fig. 3. In vitro adipocyte-cancer cell crosstalk leads to adipocyte "dedifferentiation". (A) Schemes of the co-culture model which allows the passage of soluble factors but devoid of heterotypic cell-cell contact. (B) Adipocytes co-cultured in the presence of breast cancer cells $\left(A d_{B C C}\right.$ ) show delipidation and a more elongated fibroblast-like phenotype compared with adipocytes cultured alone $(A d)$. The alterations observed in $A d_{B C C}$ are similar to those observed in vivo in CAAs at the invasive front of breast tumors (see Figure 2). Lipid droplets are stained in red using Oil red. 
A

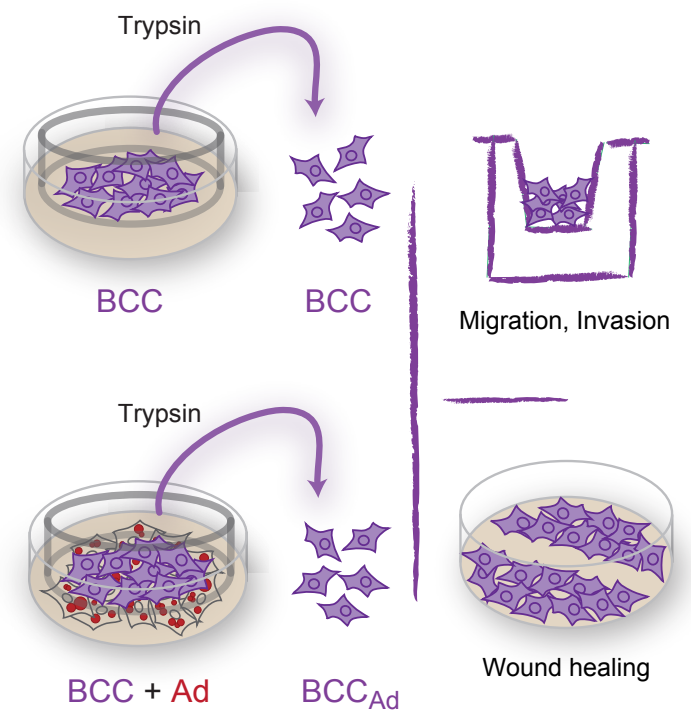

B

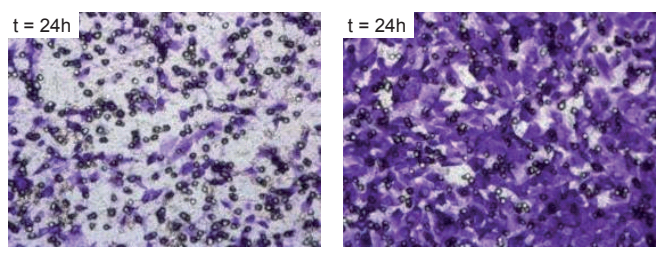

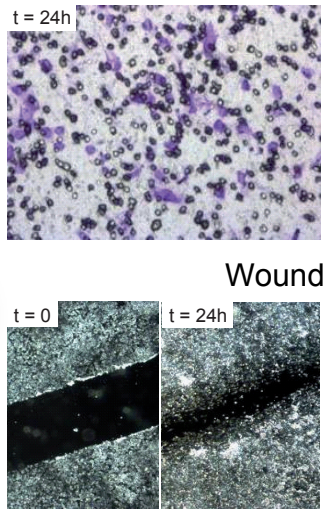

$\mathrm{BCC}$
Fig. 4. In vitro adipocyte-cancer cell crosstalk increases cancer cell aggressiveness. (A) Schemes of the coculture experiments. Breast cancer cells cultured in the presence $\left(B C C_{A d}\right)$ or in the absence (BCC) of adipocytes were further used to study their aggressive properties (migration, invasion, wound healing). (B) Photographs show cells penetrated through the membrane and present to its lower surface. $B C C_{A d}$ show increased cancer cell migration and invasion compared with $B C C$; wound healing is also higher for $B C C_{A d}$ than $B C C$. $t=$ experiment timing in hours.

invasion (lyengar et al., 2003).

Interestingly, similar effects have been reported using other types of cancer cells. Adipocytes have been shown to promote the proliferation of colon and prostate cancer cells, indicating that this phenomenon is not limited to breast cancers but is a general event (Rio, 2011).

Collectively these data indicate that in vitroadipocyte-cancer cell crosstalk leads to the production of soluble factors that increase cancer cell aggressiveness.

\section{Molecular characterisation of the adipocyte-cancer cell crosstalk}

To date, the molecular mechanisms responsible for the pejorative impact by in vivo/in vitro CAA/adipocyte-cancer cell heterotypic crosstalk have remained largely unknown. However, it might be hypothesized that they include paracrine pathways acting in both directions - from invasive cancer cells to adipocytes/CAAs, and from adipocytes/CAAs to cancer cells -. Moreover, adipocyte- or cancer cell-specific autocrine pathways might also be activated. The few results to date indicate that various biological processes are affected, including tissue remodeling, adipogenesis/energy metabolism, oncogenesis, inflammation and immune response.

\section{ECM remodeling-related factors}

There is some evidence that adipocyte ECM and ECM-related enzymes are modified, which is consistent with the dynamic modification in CAA morphology. Matrix metalloproteinase (MMPs) are enzymes specialized in ECM remodeling. They have been shown
Invasion

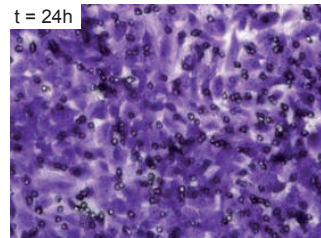

healing

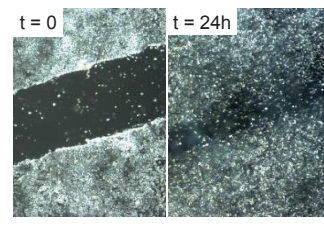

to be involved at several steps of cancer development, and represent markers of poor prognosis in breast cancer (Basset et al., 1990; Jodele et al., 2006; Kessenbrock et al., 2010). MMPs are mainly paracrine factors which are expressed and secreted by connective tissue cells. Immunohistochemical analysis of the breast cancer invasive front shows that MMP11 (also named stromelysin-3) is expressed by CAAs at the proximity of invading cancer cells. In contrast, normal resting mature adipocytes are devoid of MMP11. Thus, cancer cells induce MMP11 expression in CAAs (Fig. 5). Interestingly, MMP11 has been shown to play a role in adipogenesis since it decreases pre-adipocyte differentiation. Moreover, in vitro addition of active MMP11 recombinant protein to the culture medium of mature adipocytes induces their "de-differentiation" (Andarawewa et al., 2005). Collectively, these data indicate that the negative regulatory function on adipogenesis of MMP11 is aberrantly restored during the early steps of local tumor invasion. MMP11 therefore participates in the accumulation of fibroblast-like cells that might in reality be pre-adipocytes or de-differentiated adipocytes, since these cells are morphologically indistinguishable from fibroblasts. Accordingly, at later points of invasive carcinoma development when the constituted stroma no longer contains adipocytes, MMP11 is restricted to a particular subpopulation of fibroblast-like cells that are not myofibroblasts (Fig. 5) (Andarawewa et al., 2005). Interestingly, the concept of "cancer cell-imprinted" fibroblasts which express MMP11 has been proposed in studies using CAFs and cancer cells isolated from human breast tumors (Wang and Tetu, 2002). Consistently, in the in vitro model described above, adipocytes co-cultured with cancer cells $\left(\mathrm{Ad}_{\mathrm{BCC}}\right)$ exhibit an activated phenotype marked by MMP11 overexpression (Dirat et al., 2011)(our unpublished results). In addition to MMP11, MMP1 and MMP10 expression are also upregulated but by cancer cells $\left(\mathrm{BCC}_{\mathrm{Ad}}\right)$ (lyengar et al., 2003) (Kim et al., 2008). It cannot be excluded that some other MMPs might similarly be involved in adipocyte-cancer cell crosstalk as several MMPs contribute to adipogenesis as either positive or negative regulators (Andarawewa and Rio, 2008). Finally, the enzymatic plasminogen system is also involved, since the inhibitor of plasminogen activator (PAl1) is overexpressed in adipocytes $\left(\mathrm{Ad}_{\mathrm{BCC}}\right)$.

One of the ECM components that is altered is type VI collagen, a specific member of the soluble collagen family with predominant expression in the adipose tissue. Type VI collagen was shown to be up-regulated in adipocytes during tumorigenesis and to be critical for tumor progression due to effects of a alpha 3 chain C-terminal 
fragment (lyengar et al., 2005). Interestingly, it has recently been shown that MMP11 cleaves the native alpha 3 chain of collagen VI. This MMP11-specific collagenolytic activity is functional during fat tissue ontogenesis as well as during the cancer invasive steps (Motrescu et al., 2008). Laminin and alpha 2 integrin have also been shown to be overexpressed by cancer cells following adipocyte-cancer cell crosstalk (lyengar et al., 2003).

\section{Adipogenesis-related factors}

Adipocytes might locally provide growth/survival support to cancer cells via the secretion of various adipokines, growth factors and cytokines (Maeda et al., 1997). The sequential morphological modification of CAAs from mature adipocytes to fibroblast-like cells implies lipolysis and a defect in canonical adipocyte-differentiating factors. Consistently, adipocytes co-cultured with cancer cells exhibit decreased mRNA levels for PPAR $\gamma, a P 2, C / E B P \alpha$, resistin and hormone-sensitive lipase (HSL) (Dirat et al., 2011). Moreover, the in vitro recombinant MMP11-induced adipocyte "de-differentiation" is also associated with the downregulation at the transcriptional level of PPAR $\gamma$ and aP2 (Andarawewa et al., 2005). Moreover, CAAs have been shown to express abnormal levels of growth factors (IGF1, IGFBPs) and adipokines (leptin and adiponectin). There is increasing evidence that adiponectin and leptin, secreted by peritumor adipose tissues in several cancers including breast, are important (Miyoshi et al., 2006; Schaffler et al., 2007). Leptin appears to be a positive factor for tumor development and aggressiveness, while adiponectin protects against cancers.

\section{Immune response and inflammation-related factors}

A study profiling gene expression of breast cancer cells treated with adipocyte culture medium showed induction of several immune system-related genes (Kim et al., 2008). In vitro activated adipocytes co-cultured with cancer cells $\left(\mathrm{Ad}_{\mathrm{BCC}}\right)$ also overexpress pro-inflammatory cytokines (IL-6, IL-1 $\beta$ ) (Dirat et al., 2011). Interestingly, it has been reported that the increase of migration and invasion of estrogen receptor (ER)-negative breast cancer cells is dependent on adipocyte-secreted IL-6 (Walter et al., 2009).

\section{Oncogenesis-related factors}

Analysis of "adipocyte-primed" cancer cells $\left(\mathrm{BCC}_{\mathrm{Ad}}\right)$ show several modifications in gene expression patterns. The altered genes have been shown to be involved in various cellular functions but all are known to contribute to tumor development/progression.
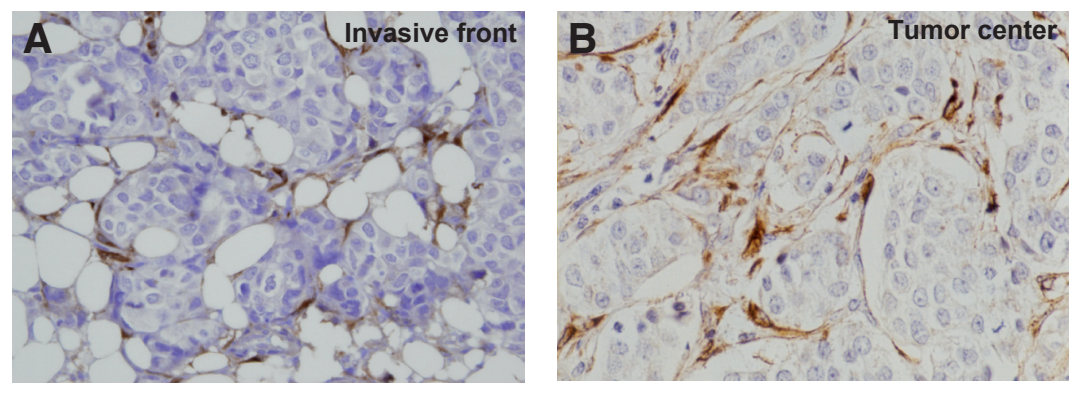

Fig. 5. Matrix metalloproteinase 11 (MMP11) immunohistochemistry of a breast carcinoma. (A) At the invasive front, MMP11 immunostaining shows that cancerassociated adipocytes (CAAs) express MMP11 (brown staining); (B) At the tumor center, numerous cancer-associated fibroblasts (CAFs) also express MMP11.
Thus, cyclin D1, NFkB, cFos, ATF3, SOX9, A20 and IGF2 are overexpressed while E-cadherin is down-regulated (lyengar et al., 2003) (Dirat et al., 2011).

Thus, resident mammary gland adipocytes play an essential role during the first steps of the tumor desmoplastic response. Adipocyte/CAA-cancer cell crosstalk induces dramatic changes in the production of molecules able to potentially impact on cancer cell survival, proliferation and differentiation as well as invasive properties (Fig. 6).

\section{Conclusion}

Collectively, these data support the concept that crosstalk with adipocytes confers a selective advantage to invasive cancer cells. Adipocytes therefore participate in a kind of vicious tumor progression cycle initiated by the invasive cancer cells (Fig. 6) (Motrescu and Rio, 2008). Although some clues have started to emerge, most of the molecular mechanisms involved remain to be determined.

\section{Do adipocyte/metabolic disorders impact on breast cancer development/progression?}

\section{Epidemiological evidence that obesity and insulin resistance/ diabetes are detrimental for breast cancer patients}

From the studies described above, an obvious concern is whether modifications in mammary resident adipocytes influence breast health. Normal adipocyte size heterogeneity is lost in obesity, and adipocyte hypertrophy (increase in cell size) often precedes hyperplasia (increase in cell number). Adipocyte hypertrophy results from excess triglyceride accumulation in existing adipocytes. Hyperplasia, referred to adipogenesis, results from the recruitment of new adipocytes from precursor cells in the adipose tissue, and involves the proliferation and differentiation of these pre-adipocytes (Gregoire, 2001). Several epidemiological studies have reported that large amounts of adipose tissues are closely associated with poor prognosis for breast cancer in obese post-menopausal women. It was proposed that elevated numbers of hypertrophied adipocytes might promote aggressive hormone-dependent breast cancers by increasing hormone synthesis (Manabe et al., 2003). Indeed, it is established that breast adipose tissue is an important site of estrogen production in post-menopausal women, and elevated estrogen biosynthesis from intratumor adipose tissue has been demonstrated (Kamat etal., 2002). This is dependent on aromatase, a key enzyme that is involved in peripheral estrogen aromatisation in the adipose tissue (Macedo et al., 2009). Moreover, several findings have indicated that mature human adipocytes possess estrogen receptors (ER), and that adipose tissue might itself be an estrogen-responsive tissue (Cooke and Naaz, 2004). However, more recent studies show that the poor prognosis for obese people with breast cancer is independent of menopause status, tumor stage, and tumor hormone status (Majed et al., 2008). Interestingly, emerging evidence suggest that host factors contribute to the occurrence of aggressive tumors in obese women, as defined by advanced stage, high grade and propensity to metastasize (Rose and Vona-Davis, 2009). In addition, it has been shown that hyperinsulinemia and type 2 diabetes are independent risk factors for poor prognosis in women with breast cancers (Duggan et al., 2010) (Gouveri et al., 2011). 


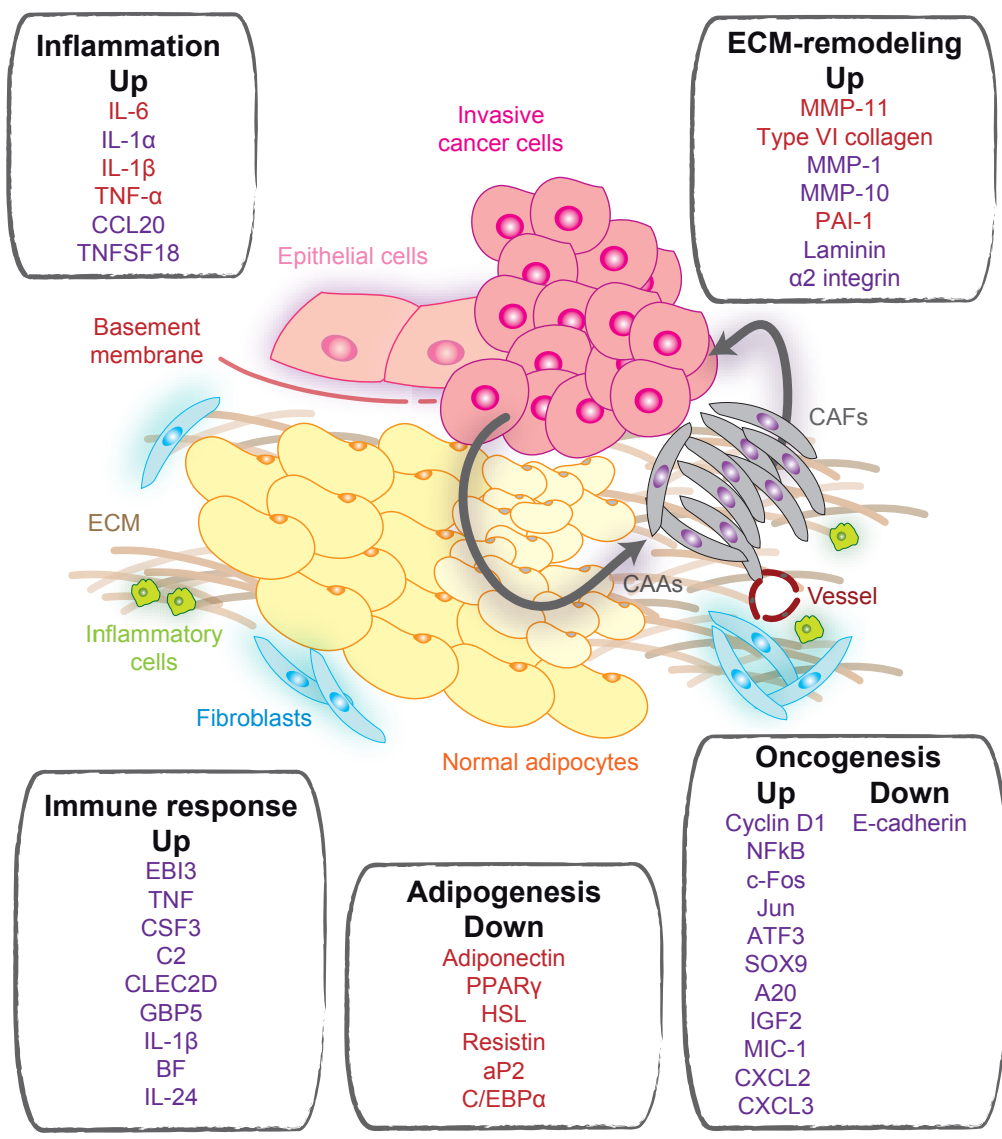

Fig. 6. Cancer-associated adipocyte (CAA)-cancer cell crosstalk orchestrates a vicious cycle promoting cancer progression. In this scenario, illegitimate crosstalk between invading cancercells and resident adipocytes and/or pre-adipocytes induces the de-differentiation of CAAs and/or prevents pre-adipocyte differentiation, leading to the accumulation/maintenance of CAFs. In turn, CAFs promote tumor progression. These events are sustained by expression/secretion of various factors by both cell types, most of which remain unidentified. Molecules whose expression have been shown to be modified at the transcriptional level following adipocyte/CAA-cancer cell crosstalk either in vitro or in vivo are mentioned in boxes (red, expressed by $A d_{B C C}$ CAAs; violet, expressed by $B C C_{A d}$ /cancer cells; up, overexpression; down, lower expression). Their biological functions have been arbitrarily subdivided into 5 sets related to inflammation, immune response, ECM remodeling, oncogenesis and adipogenesis. aP2: Adipocyte fatty acid binding protein; ATF3: activating transcription factor; BF: B factor; C/EBP $\alpha$ : CCAAT-enhancer-binding protein $\alpha ; C 2$ : complement component 2; CCL20: chemokine (C-C) motif ligand 20; CLEC2D: C-type lectin superfamily 2, member D; CSF3: colony stimulating factor 3 ; $C X C L$ : chemokine $(C-X-C$ motif) ligand; EBI: Epstein-barr virus induced gene; GBP5: guanylate binding protein 5; HSL: hormone-sensitive lipase; IGF2: insulin-like growth factor 2; IL: interleukin; MIC-1: macrophage inhibitory cytokine-1; MMP: matrix metalloproteinase; NF-KB: nuclear factor-kappa B; PAI-1: plasminogen activator inhibitor-1; PPARy: Peroxysomeproliferator activated receptor $\gamma$; SOX9: SRY-related HMG box 9; TNF: tumor necrosis factor; TNFSF18: tumor necrosis factor superfamily, member 18.

Finally, weight gain in breast cancer survivors has been shown to be associated with adverse health consequences (Duggan et al., 2010).

The pejorative effects of obesity are not restricted to breast cancer. Solid epidemiological data support the role of fat mass/ distribution in the development of risk factors, morbidity and mortality in several types of cancers (Schaffler et al., 2007; Fair and Mont- gomery, 2009; Rio, 2011). A large american prospective analysis of the weight-cancer relationship has shown that excess body mass index (BMI) is the cause of approximately $14 \%$ of all cancer deaths in men and $20 \%$ in women aged 50 years or older (Calle et al., 2003). The International Agency for Research on Cancer (IARC) has concluded that there is sufficient evidence of a cancer-preventive effect for avoiding weight gain in several cancers (Vainio et al., 2002). A similar conclusion was drawn from a Shanghai Breast Cancer Study for breast cancer (Malin et al., 2005).

The main conclusions from epidemiological studies are that obesity as well as insulin resistance/diabetes influence cancer risk, tumor behavior and clinical outcome. Excess of fat tissues is detrimental for cancer patients regardless organ. There is therefore great interest in understanding the mechanisms by which adipocytes contribute to carcinogenesis, since obesity/metabolic disorders are worldwide epidemic diseases.

\section{"Obese" adipocytes and cancer cell-primed cancer- associated adipocytes (CAAs) show similar molecular modifications}

The clinical data call into question the potential molecular impact of excess of hypertrophied resident adipocytes on breast cancer development/progression. This topic is extremely difficult to explore, and very few data are available. Most concern circulating molecules. Indeed, in addition to their local function, it cannot be excluded that adipocytes/ CAAs may also act systemically. In this context, it has been shown in vitro that several molecules expressed by CAAs following crosstalk with invasive cancer cells are secreted factors that might reach the circulation (see Fig. 6 ). Accordingly, breast cancer patients devoid of adipocyte/metabolic disorders exhibit molecular modifications in the blood, indicating that CAAs act via systemic route. In obese patients with breast cancer, we might expect obesity-dependent local and systemic effects to synergize with CAA effects. Indeed, the cellular homeostasis and the secretory profiles of hypertrophied adipocytes from cancer-free obese people are altered and increasingly dysregulated compared with normal adipocytes. Further, excess of adipose tissues located elsewhere in the body might also impact on tumor behavior via a systemic route. For example, obesity is associated with an altered cytokine profile, characterized by a reduction in the release of anti-inflammatory factors and an increase in the secretion of pro-inflammatory factors. Consequently, obesity is considered an important source of chronic inflammation (Hoene and Weigert, 2008). Moreover, in obesity, the adipose tissue is infiltrated with increased numbers of TNF $\alpha$ - and IL6-secreting macrophages (Weisberg et al., 2003). Leptin is also activated by obesity-related stimuli in adipocytes while adiponectin is decreased (Schaffler et al., 2007)(Kaur and Zhang, 2005). It is known that expanding adipose tissues require local active angiogenesis and neovascularization (Hausman and Richardson, 2004). Moreover, there are adipocyte dysfunctions linking obesity to insulin resistance and type 2 diabetes (Guilherme et al., 2008)(Rabe et al., 2008). In addition, hyperinsulinemia promotes the synthesis and biological activity of 
insulin-like growth factor (IGF1) which regulates cell proliferation (Pollak, 2008). Finally, adipose tissues also impacts the hormonal status since insulin affects the synthesis and the bioavailability of male and female sex steroids (Fischer-Posovszky et al., 2007). Interestingly, there are some molecular similarities between the blood alterations observed in breast cancer patients devoid of adipocyte/metabolic disorders and those observed in cancer-free obese people. Several of these factors have been shown to be modified in CAAs (see above Fig. 6).

Thus, several molecular alterations commonly found in adipocytes of people suffering obesity/metabolic disorders are similar to those detected in CAAs of invasive tumors. It is therefore tempting to speculate that whereas in people devoid of any adipocyte/metabolic disorders, factors that favor tumor progression are provided subsequently to stimuli initiated by CAA-cancer cell crosstalk, in obese people, this permissive microenvironment for invasive cancer cells is permanent due to stimuli coming from the plethora of hypertrophied resident adipocytes. These optimal conditions might therefore boost cancer cell aggressiveness.

\section{Adipocytes as "fuel" for cancer cells}

Although metabolic changes do not cause malignancy, they are necessary for tumor progression. Indeed, cancer cells need to proliferate rapidly and therefore use a high rate of energy-consuming processes to drive increased protein and DNAsynthesis (Menendez and Lupu, 2007). There is also a need to maintain a constant supply of lipids and lipid precursors to fuel membrane production in the highly proliferating cancer cell population. The data reported above question the possible direct involvement of CAAs in these events. Interestingly, it has been reported that epithelial cells within the mouse mammary gland are able to utilize fatty acids sequestered from fat pad adipocytes (Hovey and Aimo, 2010). In this case, epithelial cells clearly induce lipolysis in adjacent adipocytes during lactation, presumably through the action of epithelium-derived lipoprotein lipase. In turn, these adipocyte-derived fatty acids can be utilized for de novo fatty acid synthesis by lactating mammary epithelial cells. It might therefore be hypothesized that this interaction could be aberrantly restored between invasive cancer cells and resident adipocytes. Delipidation in CAAs supports such a process. Moreover, in a mouse model, Raman microscopy revealed intracellular lipid accumulation in cancer cells. Lipid-rich cancer cells are linked to cancer metastases (Le et al., 2009). Free fatty acids (FFAs) might be used for the production of signalling lipids, converted to specific lipid species for incorporation into membranes or used for mitchondrial $\beta$-oxidation to produce ATP. Although it is thought that the bioenergetic needs of cancer are predominantly met through glycolysis, the oxidation of FFAs offers an interesting alternative. Indeed, it has been shown that an increase in intracellular FFAs enhances tumor progression potentially through $\beta$-oxidation (Nomura et al., 2010).

There is a clear link between deregulated energy processing and increased cancer aggressiveness. Emerging evidence suggest that the "transfer" of lipids between adipocytes and cancer cells might represent a key energetic source for cancer cells and as such stimulate cancer progression. In this context, having an excess of "obese" adipocytes could obviously provide more triglycerides and more available FFAs to the developing tumor than normal adipocytes.

\section{Conclusion}

Improving knowledge of adipocyte/CAA functions might help to decipher the relationship between high BMI, or type 2 diabetes, and increased cancer risk and poor clinical outcome. In this context, modulating the plasticity of adipocytes and elucidating their extracellular and intracellular signaling pathways are major challenges for future research in this field. Molecules linked to adipocyte biology might offer new therapeutic opportunities for treating cancer. These data justify work aimed at exploring the feasibility of current metabolic syndrome and associated disorder treatments for anti-cancer therapy (Gouveri et al., 2011). For example, recent studies provide clues that metformin, a drug usually given for the treatment of type 2 diabetes, is associated with reduced incidence and improved prognosis of various cancers including breast cancers (Ben Sahra et al., 2010).

\section{General conclusion}

The present review provides new insights into the function of resident adipocytes in tumors. As outlined above, adipocytes within mammary gland carcinomas are clearly dynamic cells that contribute to human breast cancer progression. Its deleterious function is initiated through crosstalk with cancer cells. This review strongly supports the more general concept that the loss of adipose tissue homeostasis favors tumor aggressive behavior. In this context, it provides insights into the relationship between obesity/diabetes and cancer. This is of particular interest since recent epidemiological studies have identified obesity as one of the major risk factors for cancer. Moreover, several important considerations arise from such data, notably concerning the possibility of promoting cancer recurrence by "activating" residual cancer cells in post-mastectomy reconstruction with autologous fat transfer, or dormant malignant cells or microtumors in breast augmentation.

The cellular and molecular processes underlying adipocyte function in cancer is just beginning to unfold. Many questions remain unanswered, and it is important to further study mechanisms and signaling pathways involved to understand how adipocytes promote tumor progression. One of the most important questions to be addressed concerns the possible role of resident adipocytes in the distant tissues colonized by metastatic cells. Indeed, phenomenons similar to those observed at the primary tumor sites might be expected to exist at the secondary tumor sites. In this context, it should be noted that the microenvironment of preferential organs for metastasis development often contains adipocytes/ lipids (Siclari et al., 2006).

This research might lead in fine to the identification of adipocyterelated targets to improve diagnosis and prognosis, and/or to design innovative anticancer strategies.

\section{Acknowledgements}

We thank Susan Chan for helpful discussion. This work was supported by funds from the Institut National de la Santé et de la Recherche Médicale, the Centre National de la Recherche Scientifique, the Hôpital Universitaire de Strasbourg, the Association pour la Recherche sur le Cancer, the Ligue Nationale Française Contre le Cancer and the Comités du Haut-Rhin, du Bas-Rhin et de la Haute-Savoie (Equipe labellisée LIGUE 2010) and the Institut National du Cancer (Contrat PL-BIO ADIPOK 2011-2013). J.T. was a recipient of a Chinese fellowship (Department of Endocrine and Breast Surgery, the firstAffiliated Hospital of Chongqing Medical University, Chongq- 
ing 400016, China); E. B. was a recipient of LNCC and INCA fellowships.

\section{References}

ANDARAWEWA KL, RIO MC (2008) New insights into MMP function in adipogenesis. In The cancer degradome (Ed. DR Edwards), Springer Science, pp 353-364.

ANDARAWEWA KL, MOTRESCU ER, CHENARD MP, GANSMULLER A, STOLL I, TOMASETTO C, RIO MC (2005) Stromelysin-3 is a potent negative regulator of adipogenesis participating to cancer cell-adipocyte interaction/crosstalk at the tumor invasive front. Cancer Res 65: 10862-10871.

BASSETP, BELLOCQJP, WOLFC, STOLLI, HUTIN P, LIMACHER JM, PODHAJCER OL, CHENARD MP, RIO MC, CHAMBON P (1990) Anovel metalloproteinase gene specifically expressed in stromal cells of breast carcinomas. Nature 348: 699-704.

BEN SAHRA I, LE MARCHAND-BRUSTEL Y, TANTI JF, BOST F (2010) Metformin in cancer therapy: a new perspective for an old antidiabetic drug? Mol Cancer Ther 9: 1092-1099.

CALLE EE, RODRIGUEZC, WALKER-THURMOND K, THUN MJ (2003) Overweight, obesity, and mortality from cancer in a prospectively studied cohort of U.S. adults. N Engl J Med 348: 1625-1638.

COOKE PS, NAAZ A (2004) Role of estrogens in adipocyte development and function. Exp Biol Med (Maywood) 229: 1127-1135.

DIRAT B, BOCHET L, DABEK M, DAVIAUD D, DAUVILLIER S, MAJED B, WANG YY, MEULLE A, SALLES B, LE GONIDEC S, GARRIDO I, ESCOURROU G, VALET P, MULLER C (2011) Cancer-associated adipocytes exhibit an activated phenotype and contribute to breast cancer invasion. Cancer Res. 71:2455-2465.

DUGGAN C, IRWIN ML, XIAO L, HENDERSON KD, SMITH AW, BAUMGARTNER RN, BAUMGARTNERKB, BERNSTEINL, BALLARD-BARBASHR, MCTIERNAN A (2010) Associations of insulin resistance and adiponectin with mortality in women with breast cancer. J Clin Oncol 29: 32-39.

EDWARDS PA (2000) The use of transplanted mammary gland to study cancer signalling pathways. Adv Exp Med Biol 480: 163-167.

ELLIOTT BE, TAM SP, DEXTER D, CHEN ZQ (1992) Capacity of adipose tissue to promote growth and metastasis of a murine mammary carcinoma: effect of estrogen and progesterone. Int J Cancer 51: 416-424.

FAIR AM, MONTGOMERY K (2009) Energy balance, physical activity, and cancer risk. Methods Mol Biol 472: 57-88.

FISCHER-POSOVSZKY P, WABITSCH M, HOCHBERG Z (2007) Endocrinology of adipose tissue - an update. Horm Metab Res 39: 314-321.

GOUVERI E, PAPANAS N, MALTEZOS E (2011) The female breast and diabetes. The breast 20: 205-211.

GREGOIRE FM (2001) Adipocyte differentiation: from fibroblast to endocrine cell. Exp Biol Med (Maywood) 226: 997-1002.

GUILHERME A, VIRBASIUS JV, PURI V, CZECH MP (2008) Adipocyte dysfunctions linking obesity to insulin resistance and type 2 diabetes. Nat Rev Mol Cell Biol 9: 367-377.

HOENE M, WEIGERT C (2008) The role of interleukin-6 in insulin resistance, body fat distribution and energy balance. Obes Rev 9: 20-29.

HOVEY RC, AIMO L (2010) Diverse and active roles for adipocytes during mammary gland growth and function. J Mammary Gland Biol Neoplasia 15: 279-290.

IYENGAR P, COMBS TP, SHAH SJ, GOUON-EVANS V, POLLARD JW, ALBANESE C, FLANAGAN L, TENNISWOOD MP, GUHA C, LISANTI MP, PESTELL RG, SCHERER PE (2003) Adipocyte-secreted factors synergistically promote mammary tumorigenesis through induction of anti-apoptotic transcriptional programs and proto-oncogene stabilization. Oncogene 22: 6408-6423.

IYENGARP, ESPINAV, WILLIAMS TW, LINY, BERRYD, JELICKS LA, LEEH, TEMPLE K, GRAVES R, POLLARD J, CHOPRA N, RUSSELL RG, SASISEKHARAN R, TROCK BJ, LIPPMAN M, CALVERT VS, PETRICOIN EF, 3RD, LIOTTA L, DADACHOVA E, PESTELL RG, LISANTI MP, BONALDO P, SCHERER PE (2005) Adipocyte-derived collagen $\mathrm{VI}$ affects early mammary tumor progression in vivo, demonstrating a critical interaction in the tumor/stroma microenvironment. J Clin Invest 115: 1163-1176.

JODELE S, BLAVIER L, YOON JM, DECLERCK YA (2006) Modifying the soil to affect the seed: role of stromal-derived matrix metalloproteinases in cancer progression. Cancer Metastasis Rev 25: 35-43.

KAMATA, HINSHELWOOD MM, MURRY BA, MENDELSON CR (2002) mechanisms in tissue-specific regulation of estrogen biosynthesis in humans. Trends Endocrinol Metab 13: 122-128.

KAUR T, ZHANG ZF (2005) Obesity, breast cancer and the role of adipocytokines. Asian Pac J Cancer Prev 6: 547-552.

KESSENBROCK K, PLAKS V, WERB Z (2010) Matrix metalloproteinases: regulators of the tumor microenvironment. Cell 141: 52-67.

KIM JH, KIM KY, JEON JH, LEE SH, HWANG JE, LEE JH, KIM KK, LIM JS, KIM $\mathrm{KI}$, MOON EY, LEE HG, RYU JH, YANG Y (2008) Adipocyte culture medium stimulates production of macrophage inhibitory cytokine 1 in MDA-MB-231 cells. Cancer Lett 261: 253-262.

KIMIJIMA I, OHTAKE T, SAGARA H, WATANABE T, TAKENOSHITA S (2000) Scattered fat invasion: an indicator for poor prognosis in premenopausal, and for positive estrogen receptor in postmenopausal breast cancer patients. Oncology 59 Suppl 1: 25-30.

LE TT, HUFF TB, CHENG JX (2009) Coherent anti-Stokes Raman scattering imaging of lipids in cancer metastasis. BMC Cancer 9: 42.

LEFEBVRE O, WOLF C, LIMACHER JM, HUTIN P, WENDLING C, LEMEUR M, BASSET P, RIO MC (1992) The breast cancer-associated stromelysin-3 gene is expressed during mouse mammary gland apoptosis. J Cell Biol 119: 997-1002.

MACEDO LF, SABNIS G, BRODIE A (2009) Aromatase inhibitors and breast cancer. Ann N Y Acad Sci 1155: 162-173.

MAEDA K, OKUBO K, SHIMOMURA I, MIZUNO K, MATSUZAWA Y, MATSUBARA K (1997) Analysis of an expression profile of genes in the human adipose tissue. Gene 190: 227-235.

MAJED B, MOREAU T, SENOUCI K, SALMON RJ, FOURQUET A, ASSELAIN B (2008) Is obesity an independent prognosis factor in woman breast cancer? Breast Cancer Res Treat 111: 329-342.

MALIN A, MATTHEWS CE, SHU XO, CAI H, DAI Q, JIN F, GAO YT, ZHENG W (2005) Energy balance and breast cancer risk. Cancer Epidemiol Biomarkers Prev 14: 1496-1501.

MANABE Y, TODA S, MIYAZAKI K, SUGIHARA H (2003) Mature adipocytes, but not preadipocytes, promote the growth of breast carcinoma cells in collagen gel matrix culture through cancer-stromal cell interactions. J Pathol 201: 221-228.

MENENDEZ JA, LUPU R (2007) Fatty acid synthase and the lipogenic phenotype in cancer pathogenesis. Nat Rev Cancer 7: 763-777.

MIYOSHI Y, FUNAHASHI T, TANAKA S, TAGUCHI T, TAMAKI Y, SHIMOMURA I, NOGUCHI S (2006) High expression of leptin receptor mRNA in breast cancer tissue predicts poor prognosis for patients with high, but not low, serum leptin levels. Int J Cancer 118: 1414-1419.

MOTRESCUER, RIO MC (2008) Cancer cells, adipocytes and matrix metalloproteinase 11: a vicious tumor progression cycle. Biol Chem 389: 1037-1041.

MOTRESCU ER, BLAISE S, ETIQUE N, MESSADDEQ N, CHENARD MP, STOLL I, TOMASETTO C, RIO MC (2008) Matrix metalloproteinase-11/stromelysin-3 exhibits collagenolytic function against collagen VI under normal and malignant conditions. Oncogene 27: 6347-6355.

NAKAJIMA I, MUROYA S, TANABE R, CHIKUNI K (2002) Positive effect of collagen $\mathrm{V}$ and $\mathrm{VI}$ on triglyceride accumulation during differentiation in cultures of bovine intramuscular adipocytes. Differentiation 70: 84-91.

NOMURA DK, LONG JZ, NIESSEN S, HOOVER HS, NG SW, CRAVATT BF (2010) Monoacylglycerol lipase regulates a fatty acid network that promotes cancer pathogenesis. Cell 140: 49-61.

POLLAK M (2008) Insulin and insulin-like growth factor signalling in neoplasia. Nat Rev Cancer 8: 915-928.

RABE K, LEHRKE M, PARHOFER KG, BROEDL UC (2008) Adipokines and insulin resistance. Mol Med 14: 741-751.

RIO MC (2011) The role of Cancer-Associated Adipocytes (CAA) in the dynamic interaction between the tumor and the host: Springer SBM.

RONNOV-JESSEN L, PETERSEN OW, KOTELIANSKY VE, BISSELL MJ (1995) The origin of the myofibroblasts in breast cancer. Recapitulation of tumor environment in culture unravels diversity and implicates converted fibroblasts and recruited smooth muscle cells. J Clin Invest 95: 859-873.

ROSE DP, VONA-DAVIS L (2009) Influence of obesity on breast cancer receptor status and prognosis. Expert Rev Anticancer Ther 9: 1091-1101.

SCHAFFLER A, SCHOLMERICH J, BUECHLER C (2007) Mechanisms of disease: 
adipokines and breast cancer - endocrine and paracrine mechanisms that connect adiposity and breast cancer. Nat Clin Pract Endocrinol Metab 3: 345-354.

SICLARI VA, GUISE TA, CHIRGWIN JM (2006) Molecular interactions between breast cancer cells and the bone microenvironment drive skeletal metastases. Cancer Metastasis Rev 25: 621-633.

VAINIO H, KAAKS R, BIANCHINI F (2002) Weight control and physical activity in cancer prevention: international evaluation of the evidence. Eur J Cancer Prev 11 Suppl 2:S94-100.

WALTER M, LIANG S, GHOSH S, HORNSBY PJ, LI R (2009) Interleukin 6 secreted from adipose stromal cells promotes migration and invasion of breast cancer cells. Oncogene 28: 2745-2755.

WANG CS, TETU B (2002) Stromelysin-3 expression by mammary tumor-associated fibroblasts under in vitro breast cancer cell induction. Int J Cancer 99: 792-799.

WEISBERG SP, MCCANN D, DESAI M, ROSENBAUM M, LEIBEL RL, FERRANTE AW, JR. (2003) Obesity is associated with macrophage accumulation in adipose tissue. J Clin Invest 112: 1796-1808.

YAMAGUCHI J, OHTANI H, NAKAMURA K, SHIMOKAWA I, KANEMATSU T (2008) Prognostic impact of marginal adipose tissue invasion in ductal carcinoma of the breast. Am J Clin Pathol 130: 382-388.

\section{Further Related Reading, published previously in the Int. J. Dev. Biol.}

Growth factor-defined culture medium for human mesenchymal stem cells

Sumiyo Mimura, Naohiro Kimura, Mitsuhi Hirata, Daiki Tateyama, Midori Hayashida, Akihiro Umezawa, Arihiro Kohara, Hiroki Nikawa, Tetsuji Okamoto and Miho K. Furue

Int. J. Dev. Biol. (2011) 55: 181-187

Pdx1-transfected adipose tissue-derived stem cells differentiate into insulin-producing cells in vivo and reduce hyperglycemia in diabetic mice

Hiromitsu Kajiyama, Tatsuo S. Hamazaki, Makoto Tokuhara, Shinji Masui, Koji Okabayashi, Kiyoshi Ohnuma, Shigeharu Yabe, Kazuki Yasuda, Shoichi Ishiura, Hitoshi Okochi and Makoto Asashima

Int. J. Dev. Biol. (2010) 54: 699-705

Epithelial-Mesenchymal Transitions in development and disease: old views and new perspectives

M. Angela Nieto

Int. J. Dev. Biol. (2009) 53: 1541-1547

Analysis of tenascin mRNA expression in the murine mammary gland from embryogenesis to carcinogenesis: an in situ hybridization study

I Kalembey, T Yoshida, K Iriyama and T Sakakura

Int. J. Dev. Biol. (1997) 41: 569-573

Expression of c-ets-1 and uPA genes is associated with mammary epithelial cell tubulogenesis or neoplastic scattering A Delannoy-Courdent, W Fauquette, X F Dong-Le Bourhis, B Boilly, B Vandenbunder and X Desbiens

Int. J. Dev. Biol. (1996) 40: 1097-1108

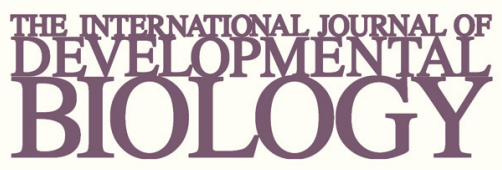

Volume 54 Nos. 6/7
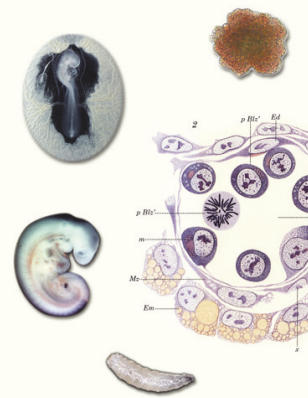

(2) $(8)$

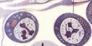

(2) (c)
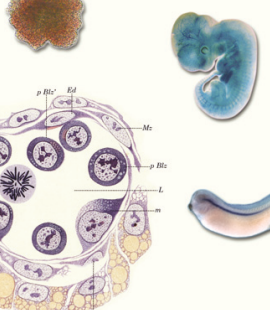

$$
\text { 政 }
$$

${ }^{L}$
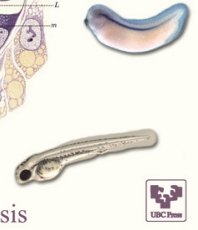

Developmental Hematopoiesis
5 yr ISI Impact Factor $(2010)=2.961$
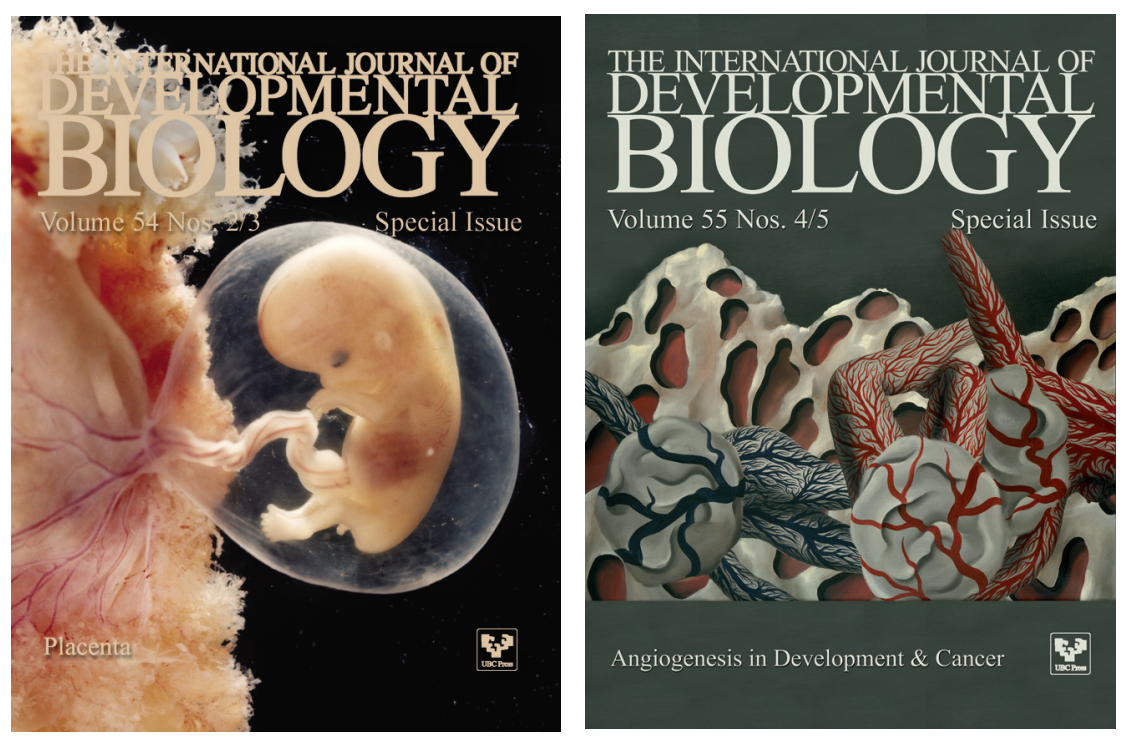